\title{
Ultrastable stimulus-response latencies: Acquisition and stimulus control
}

\author{
GORDON W. HOPKINS and ALFRED B. KRISTOFFERSON \\ McMaster University, Hamilton, Ontario, Canada
}

\begin{abstract}
Modifications were made to Kristofferson's (1976) response-stimulus synchronization procedure which resulted in a further reduction in the estimate of minimum S-R latency variance. Minimum variances near $50 \mathrm{msec}^{2}$ were obtained whether mean latency was 310 or $550 \mathrm{msec}$. Within this range, latency distributions were the same, symmetrical and sharppeaked, and unlike typical RT. All responses fell within a 50-msec time window. This independence of mean latency and latency variance was present throughout acquisition. A special technique allowed isolation of the controlling stimulus for synchronization timing, and showed that subjects were able to transfer control to another modality with no loss of performance. Results are discussed in terms of support for the notion of nonvariable, internally timed delays which can be inserted in the S-R chain. These delays are easily adjustable, but, once set, are deterministic. The role of feedback in acquisition and maintenance of synchronization performance is also examined.
\end{abstract}

Several investigators (viz, Kornblum, 1973; Ollman \& Billington, 1972; Saslow, 1974; Snodgrass, Luce, \& Galanter, 1967) have concerned themselves with isolating the "true form" of the reaction time (RT) distribution, in order to facilitate theorizing about elementary timing mechanisms underlying such behavior. The general paradigm used for approaching the so-called "true reaction time" distribution has usually involved experimentally shifting the simple RT distribution right or left along the time axis until minimum response latency variance is obtained. Shifting of distributions has been accomplished either by use of narrow payoff bands around different points following the action stimulus (Saslow, 1974; Snodgrass, 1969; Snodgrass et al., 1967) or by instructing subjects to produce a target response latency and informing them of their accuracy on each trial (Ollman \& Billingion, 1972). The basic assumption underlying the use of such procedures is that time estimation responses triggered by either the ready signal (when the distribution is shifted left) or the action stimulus (when the distribution is shifted right) are more variable than true reaction time responses triggered by the action stimulus. The goal, therefore, would be to maximize the proportion of true reaction times in the observed distribution by minimizing variance via these shifting procedures.

True reaction times are commonly thought to be the sum of three components: namely, stimulus input time, central decision time, and motor response time. Time estimates require an additional delay compo-

This research was supported by Grant A7919 from the National Research Council of Canada. We thank Joan Brewster for many valuable discussions related to this paper. nent which is assumed to contribute variability of its own which increases monotonically with its mean length. This is based on findings of proportional increase in standard deviation of response latencies as a function of the interval to be estimated (Snodgrass, Luce, \& Galanter, 1967; Woodrow, 1930, 1933).

Recently, however, Kristofferson (1976) has reported experiments which indicate that, under certain conditions, time estimation responses can be made with no more, and often less, variance than is typical of simple RT. The procedure involved a responsestimulus synchronization task similar to that employed by Naatanen, Muranen, and Merisalo (1974) for measuring peak expectancy in simple RT. Two stimuli were presented, separated by a short, fixed interval, and the subject was instructed to anticipate the second stimulus, timing from the first, and make his response in synchrony with the second stimulus. Much practice was given with the same interval, and the response was a finger press. Using such a procedure, Kristofferson (1976) found that the anticipation interval between the two stimuli could be gradually changed from values typical of simple RT (160 $\mathrm{msec}$ ) all the way to $550 \mathrm{msec}$ with no change in variance, or shape, of the response latency distribution. This led him to reject the idea that response latency variance is necessarily a monotonically increasing function of mean latency over this range and to propose the hypothesis of deterministic delays. These variance-free, adjustable delays were thought to be in the afferent latency of the stimulus. Although speculative, such a notion is consistent with certain assumptions and conclusions derived from work on successiveness discrimination (Allan \& Kristofferson, 1974; Kristofferson, 1967), duration discrimination 
(Kristofferson, 1977), temporal order discrimination (Allan, 1975), and reaction time (Ollman \& Billington, 1972).

Kristofferson (1976) reported that response latency distributions obtained using response-stimulus synchronization were all sharp-peaked, symmetrical, and well centered about the point of veridical synchrony over the entire range tested. In contrast, simple reaction time distributions are usually positively skewed, with high tails and typically more than four times the $100 \mathrm{msec}^{2}$ response latency variance reported by Kristofferson. Saslow (1974) has obtained some symmetrical RT distributions using narrow payoff bands, but minimum variances were still more than double those obtained with response-stimulus synchronization. Time-estimation response distributions are usually symmetrical, but with variance levels much higher than any of those mentioned above.

Kristofferson's (1976) findings, that the degree of temporal certainty associated with time estimation responses is independent of mean latency over a substantial range of intervals and is the same as when the response is a simple $\mathrm{RT}$, are contrary to current thinking about timing mechanisms. They indicate that added delays can be inserted into an S-R chain without any associated increase in variance. Moreover, the shape of the distribution obtained when response latency variance is minimized is very symmetrical, sharply peaked, and very similar in form despite large changes in mean.

This simple form of the distribution, especially the sharp peak, suggests the contribution of relatively few sources of variance, because if the distribution was a convolution of many component distributions a sharp peak would not be expected. Wing and Kristofferson (1973), using an interresponse timing procedure, were able to provide independent estimates of two variance components-efferent delay variance and central timing variance. Subjects' responses were similar to those used in Kristofferson's (1976) experiments but were part of a series of repeated finger taps. The obtained variance estimates for the efferent component ranged from 10 to $50 \mathrm{msec}^{2}$. Subtracting this amount from the minimum total variance of $100 \mathrm{msec}^{2}$ obtained by Kristofferson led to speculation that the central component could be represented by a triangular distribution with a base of somewhat less than $50 \mathrm{msec}$. The existence of such a unit of central temporal variability is implied by Kristofferson's (1967) time quantum theory.

The delay, as mentioned earlier, may be part of the afferent latency, and, if so, it adds no variability to the S-R chain. Kristofferson (1976) argues that if this possibility is correct, these delays could play a significant role in individual acts of information processing. However, many questions related to the existence and use of such delays in synchronization performance remain.
The present experiments seek to extend Kristofferson's (1976) findings within the range of synchronization intervals where deterministic delays appear to be utilized. Of primary interest is refinement of response-stimulus synchronization procedures so as to reduce unwanted sources of variance to a minimum and to allow full experimental manipulation of controlling stimuli.

In Kristofferson's (1976) procedure, some variability associated with recording the response could have been encountered, because the microswitch used entailed some movement time and required that a force be exerted against a spring in order for electrical contact to occur. Consequently, the synchronization response in the present procedure is changed to a finger-withdrawal response, from a touchsensitive button, which involves no movement time. Electrical contact is effected as soon as the finger breaks contact with the button.

Another procedural change is instituted to alleviate a potential problem encountered with experimenterpaced trials. In Kristofferson's procedure, trials were presented at regular intervals whether the subject was ready or not. Under these conditions, momentary lapses of attention or any other kind of disruption could affect response latencies on some trials and inflate variance measures. Consequently, experimenterpaced trials are replaced by subject-paced trials in which the presentation of the stimulus sequence is initiated by the subject.

Although the use of subject-paced trials reduces the problem of momentary inattention, it makes it difficult to determine which stimulus is controlling synchronization responding. The subject's initiation response, $R_{i}$, occurs just prior to the first auditory pulse, $P_{1}$ (see Figure 1) by the amount of a brief foreperiod. The second auditory pulse, $\mathbf{P}_{\mathbf{2}}$, follows $P_{1}$ by a fixed interval, and the subject's task is to time an appropriate interval and to trigger his synchronization response, $R_{s}$, such that it coincides with the onset of $P_{2}$. Since $R_{i}$ and $P_{1}$ occur in such close temporal proximity, some technique is required to discriminate which of these two events is the controlling stimulus for the timing of $\mathbf{R}_{s}$. The technique used involves providing the experimenter with the ability to manipulate foreperiod variability and to omit $\mathbf{P}_{1}$ from the trial sequence. Objective timing of the subject's response latencies is always made relative to the time-point $T_{0}$. Thus, when $P_{1}$ is present, it provides a good cue for synchronization timing because it is time-locked to the onset of $P_{2}$. The same is true of $R_{i}$ only if the foreperiod is constant. Therefore, with a variable foreperiod, if the subject is ignoring $P_{1}$ and timing his synchronization response from $R_{i}$, then removal of $P_{1}$ should have little effect on performance. However, if the subject is using $\mathbf{P}_{\mathbf{1}}$ for timing control, then its removal should be reflected in an increase in response 


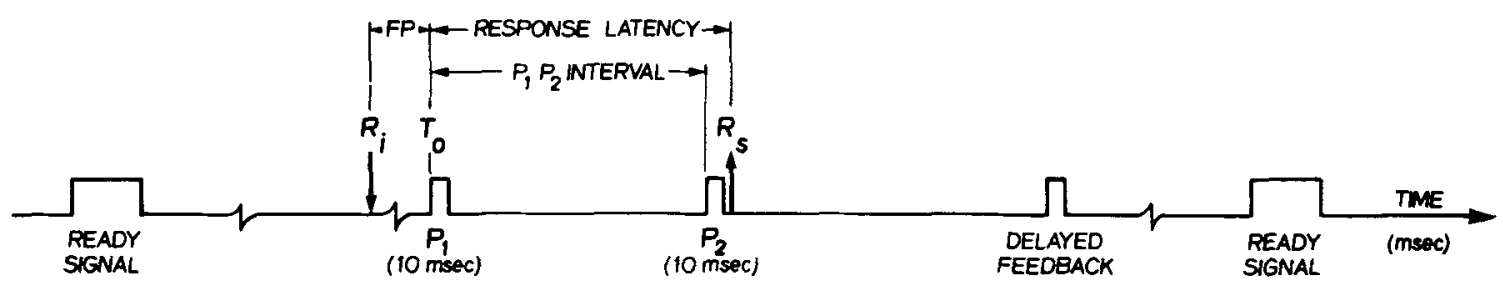

Figure 1. Diagram of a typical synchronization trial. Spacing of trials is paced by the subject's initiation response. $R_{j}$ can be made any time after the ready signal. FP refers to foreperiod duration.

latency variance by an amount equal to, if not greater than, the variability of the foreperiod. With the appropriate controls, these stimulus manipulations allow unambiguous identification of the controlling stimulus in this task.

Kristofferson's (1976) findings of new, low levels of response latency variance were mainly attributed to exhaustive training under fixed conditions and to the use of the perceptual feedback which is inherent in his procedure. Knowledge of results appeared to be important during acquisition of synchronization behavior and was provided by the perceived temporal relation between the sound of the response button, which was audible to the subject, and the auditory synchronization pulse. However, this type of perceptual feedback was very complex and difficult to specify to the subject. Consequently, the revised procedure to be used here incorporates an additional delayed feedback signal that can be well specified in instructions to subjects. Immediate perceptual feedback in the new task differs from that provided in Kristofferson's procedure because of the use of a silent switch. With no response sound, the subject has to make an evaluation of the temporal relation between the tactile stimulation produced by the finger-withdrawal response and the auditory stimulation produced by the synchronization pulse.

One of the goals to be met in estimating minimum response latency variance is to eliminate, or at least minimize, any sequential dependencies in responding because such dependencies contribute an additional, extraneous component of variance into the measurements. Since feedback is a major part of the present procedure, if subjects adopt an error correction strategy on a trial-to-trial basis, such unwanted inflation of variance might occur. Thus, analysis of temporal autocorrelation of response latencies is important both for determining the effect of feedback and for insuring accuracy of the minimum variance estimates.

Using these revised procedures, the present experiments are concerned with answering the following questions related to the remarkable human timing abilities observed by Kristofferson (1976): Can special procedures further reduce the minimum vari- ability of S-R latencies; and if so, what will the form of the distribution be? Is the variability of these centrally timed delays independent of mean delay throughout acquisition? What stimuli actually control synchronization performance? And finally, what is the nature of sequential dependencies between response latencies during and after acquisition?

\section{METHOD}

\section{Subjects}

Five graduate students, two male and three female, including the first author, participated in the experiment. Two of the subjects, C.H. and G.H., were both well practiced in reaction time and synchronization experiments.

\section{Procedure}

A response-stimulus synchronization task, similar to that used by Kristofferson (1976), was employed. All sequencing of stimuli, recording of responses, and analysis of data were under control of a Digital Equipment Corporation PDP-8e digital computer. Subjects were run one at a time in a small, sound-attenuated room. A typical trial sequence is diagrammed in Figure 1. It begins with a $500 \mathrm{msec}$ visual ready signal which indicates to the subject that a trial sequence is available. To initiate the sequence, the subject must place his index finger onto a touchsensitive button. This is referred to as the initiation response, $\mathbf{R}_{\mathrm{i}}$. The electronic switching produced by finger contact triggers a short, variable foreperiod. Foreperiod durations are selected randomly, on each trial, from a uniform distribution spanning a range of 51 to $71 \mathrm{msec}$, with a mean of $61 \mathrm{msec}$ and variance of $33.3 \mathrm{msec}^{2}$. Following the foreperiod, two $10 \mathrm{msec}$ auditory pulses, $P_{1}$ and $P_{2}$, are presented, separated by an empty interval. This interval, measured from onset to onset, is referred to as the $\mathbf{P}_{\mathbf{1}} \mathbf{P}_{\mathbf{2}}$ interval and remains constant for a given subject throughout the entire experiment.

The subject is instructed to withdraw his index finger from the touch-sensitive button in synchrony with the onset of $\mathbf{P}_{2}$. In order to accomplish this, the subject must time an appropriate interval and trigger his response at some time prior to $P_{2}$ if his overt synchronization response, $\mathbf{R}_{s}$, is to coincide with the onset of $\mathbf{P}_{\mathbf{2}}$. Response latency is measured, to the nearest millisecond, from $P_{1}$ onset $\left(T_{0}\right)$ to the moment of finger withdrawal. Error of synchrony is defined as response latency minus the $P_{1} P_{2}$ interval; thus, a negative value represents an early response. Immediate perceptual feedback is available to the subject by comparing his time of response with the time of occurrence of the synchronization pulse, $P_{2}$. Delayed feedback, indicating the subject's error of synchrony, is also provided on each trial. It consists of a third auditory pulse $500 \mathrm{msec}$ after $P_{2}$ offset. The duration of this feedback pulse is equal in magnitude to the absolute value of the error of synchrony in milliseconds while the direction of error is indicated by a brief, $100 \mathrm{msec}$ light which accompanies the feedback pulse if the subject's response occurred early-prior to $P_{2}$ onset. Following feed- 
Table 1

Chronology of Experimental Conditions

\begin{tabular}{|c|c|c|c|c|c|}
\hline \multirow[b]{2}{*}{ Condition } & \multicolumn{5}{|c|}{ Subject } \\
\hline & G.H. & G.T. & C.H. & J.B. & M.W. \\
\hline & $1-30$ & $1-30$ & & & $1-105$ \\
\hline$P_{2}$ Ónly & $31-40$ & $31-45$ & & & $106-115$ \\
\hline$P_{1} P_{2}$ & $41-45$ & $46-50$ & $1-35$ & $1-35$ & \\
\hline$P_{1}$ Ónly & $\begin{array}{l}46-55 \\
56-60\end{array}$ & $\begin{array}{l}51-60 \\
61-65\end{array}$ & $\begin{array}{l}36-45 \\
46-50\end{array}$ & $\begin{array}{l}36-45 \\
46-50\end{array}$ & \\
\hline $\begin{array}{l}P_{1} P_{2} \\
P_{2} \text { Only }\end{array}$ & $\begin{array}{l}50-00 \\
61-90\end{array}$ & $\begin{array}{l}01-03 \\
66-90\end{array}$ & $\begin{array}{l}40-50 \\
51-75\end{array}$ & $\begin{array}{l}40-50 \\
51-80\end{array}$ & \\
\hline$P_{1} P_{2}$ & $91-100$ & $91-100$ & $76-90$ & $81-125$ & \\
\hline$P_{1}$ Ónly & $101-155$ & $101-120$ & $91-105$ & $126-193$ & \\
\hline $\mathrm{P}_{1}$ Only (CFP) & $156-165$ & & & & \\
\hline$P_{1} P_{2}$ & $\begin{array}{l}166-180 \\
181-200\end{array}$ & $121-135$ & $106-125$ & & \\
\hline No $P_{1}$ or $P_{2}$ & & $136-160$ & & & \\
\hline$P_{2}$ Only & & $161-170$ & $126-135$ & & \\
\hline $\mathrm{P}_{2}$ Only (CFP) & & & $136-145$ & & $116-120$ \\
\hline 9 & 201-210 & 171-180 & $146-175$ & & \\
\hline
\end{tabular}

Note-Numbers of the sessions devoted to each experimental condition are given in the body of the table. CFP means constant foreperiod.

back, there is a fixed delay of about $2,000 \mathrm{msec}$ (depending upon the $\mathbf{P}_{1} \mathbf{P}_{2}$ interval) before the ready signal for the next trial. Some experimental manipulations alter this stimulus configuration of the typical trial. These manipulations include selective omission of one or both of the auditory pulses, $P_{1} P_{2}$, and substitution of a constant foreperiod instead of a variable one.

The auditory pulses, $P_{1}$ and $P_{2}$, are both $2,000 \mathrm{~Hz}$ sinusoids gated at zero-crossing with a rise-decay time of $2.5 \mathrm{msec}$. They are delivered binaurally over earphones at a loudness of $68 \mathrm{~dB}$ re .0002 dynes $/ \mathrm{cm}^{2}$ when on continuously. The feedback pulse is a distinctive sound produced by the mixture of 2,000 and $10,000 \mathrm{~Hz}$ sinusoids. It is slightly louder than $P_{1}$ and $P_{2}$. The responses produce no sound and electronic switching is produced instantaneously. There is no travel time associated with this type of response button. The touch-sensitive button is a small brass knob $1.2 \mathrm{~cm}$ in diameter which protrudes $.5 \mathrm{~cm}$ out of a padded armrest lying on a table. The armrest provides support for the entire forearm and the heel of the hand rests on a brass plate mounted flush into the armrest at the appropriate position. The hand must make contact with both brass plate and button for a resistance change to cause electronic switching to occur. Thus, responses consist of ballistic movements of the index finger only. Other parts of the hand and forearm remain flat against the armrest and relatively stationary. Between responses, the index finger is rested against the middle finger to reduce the chance of accidental contact with the response button.

A session consists of four blocks of 100 consecutive trials each, with a rest of $1 \mathrm{~min}$ between blocks. Length of session is dependent upon subjects' delays in initiating trials, but typically a session lasts less than $45 \mathrm{~min}$. Subjects generally run one session a day, at a fixed time, except weekends. The first block of 100 trials is considered warm-up and discarded from every session, leaving $\mathbf{3 0 0}$ trials per session for analysis. Subjects occasionally brush a finger against the response button producing an extremely aberrant response which is also discarded from analysis. However, this error is rare and generally a full $\mathbf{3 0 0}$ responses per session are analyzed. Following each session, the subject is informed of means and variances and encouraged to reduce his synchronization error and variance.

To summarize, the basic procedure differs from Kristofferson's (1976) in several ways. A finger-withdrawal response is required from a touch-sensitive button which produces no response sound. Thus, the only source for immediate perceptual feedback remaining is that provided by comparison of the temporal relation between auditory $\left(\mathrm{P}_{2}\right)$ and tactile $\left(\mathrm{R}_{\mathrm{s}}\right)$ stimulation. To supplement this, a well-specified, delayed feedback signal is added to the stimulus sequence. Finally, the trials are subject-paced with a very short foreperiod between the initiation response and the beginning of the $P_{1} P_{2}$ interval.

\section{RESULTS AND DISCUSSION}

The $P_{1} P_{2}$ intervals were fixed at $\mathbf{4 6 0} \mathrm{msec}$ for subjects G.H. and J.B., $310 \mathrm{msec}$ for subjects G.T. and C.H., and $360 \mathrm{msec}$ for subject M.W. Each subject received several stimulus manipulations during the experiment and remained on a particular condition until performance was stable or at least until a definite trend toward stability was evident. A chronology of each subject's experience with various stimulus manipulations, or conditions, used in the experiment is given in Table 1 in terms of session number.

\section{Acquisition}

All subjects started training with the full procedure as described above (both $P_{1}$ and $P_{2}$, followed by feedback). Figure 2 shows the effect of practice upon the variance of response latencies with the data grouped into blocks of 20 sessions. Points plotted represent the arithmetic average of only those sessions, within the group of 20 , which were obtained under the baseline condition-both $P_{1}$ and $P_{2}$ present. Consequently, there are some gaps where practice on intervening conditions continued over more than 20 sessions. Acquisition data for all subjects generally show a rapid decline in response latency variance over the first 20 to 40 sessions, followed by increasingly slow improvements. Naive subjects started at variances of 3,000 to $4,000 \mathrm{msec}^{2}$, but in less than 40 sessions all of them were exhibiting response latency distributions with variances lower than the lowest reported variance levels for simple RT (Saslow, 1974). Subjects C.H. and G.H. started out with lower vari- 


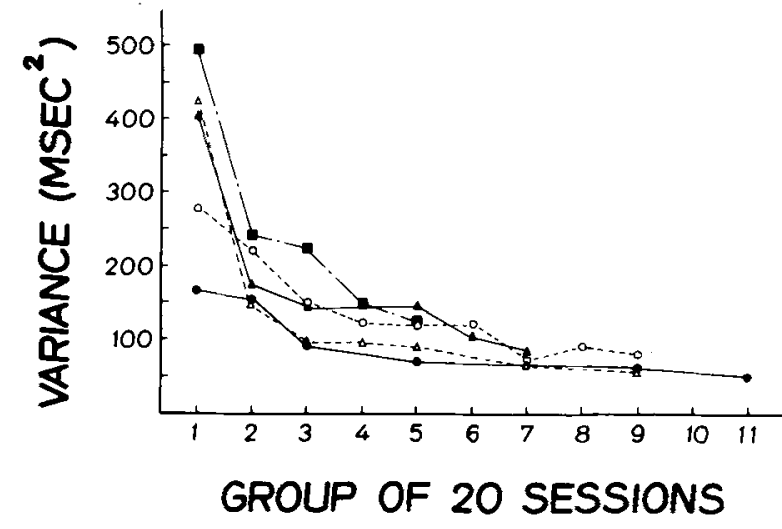

Figure 2. Decrease in mean within-block variance as a function of practice for five subjects at three different $P_{1} P_{2}$ intervals. Solid lines are for subjects G.H. (closed circles) and J.B. (closed triangles) with a $P_{1} P_{2}$ interval of $\mathbf{4 6 0}$ msec. Dashed lines are for subjects C.H. (open circles) and G.T. (open triangles) at $310 \mathrm{msec}$ and the broken line is for subject M.W. at 360 msec.

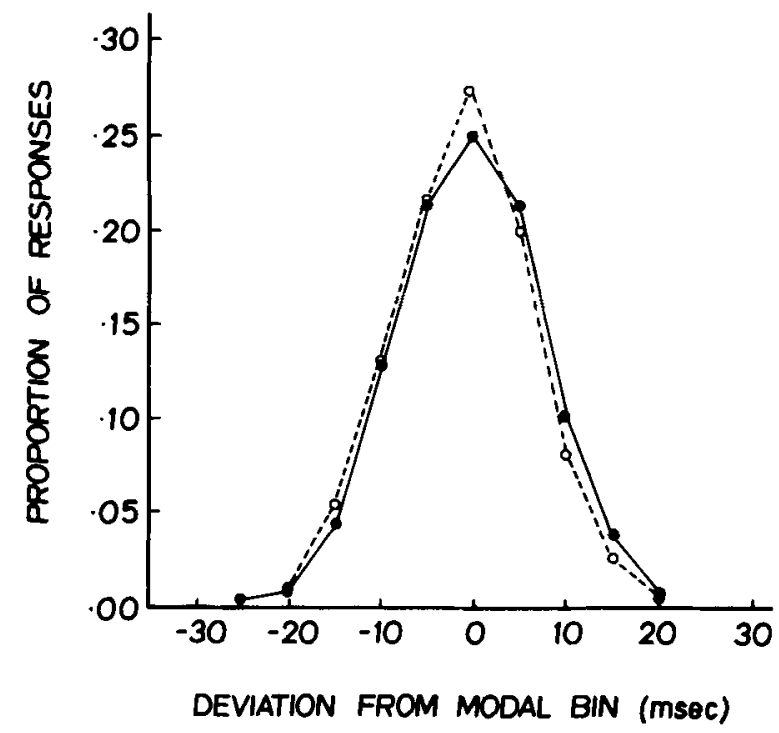

Figure 3. Superimposed relative frequency distributions, of 1,500 responses each, showing similarities in performance for two subjects at different $P_{1} P_{2}$ intervals. Solid line is for subject G.T. $(M=308$, total variance $=57$, mean within-block variance $=54, P_{1} P_{2}$ interval $=310$ ). Dashed line is for subject G.H. $(M=461$, total variance $=52$, mean within-block variance $=51, P_{1} P_{2}$ interval $\left.=460\right)$. Bin size $=5$ msec.

ances, presumably due to their prior experience, but any advantage over the other subjects was shortlived and not readily apparent after the first group of 20 sessions.

Acquisition performance, in terms of mean response latency, is not shown here because subjects' means quickly stabilized about the point of veridical synchrony over the first five sessions. By session 30 , the mean error of synchrony rarely exceeded $\pm 5 \mathrm{msec}$ and, in most cases, mean response latency was within $\pm 2 \mathrm{msec}$ of perfect synchrony, with no evidence of preferential responding, early or late, for any of the subjects.

It may be noted in Figure 2 that the acquisition curves-neglecting the first point for subjects C.H. and G.H.- show very similar time courses despite the fact that: (1) different subjects are involved, (2) three different $P_{1} P_{2}$ intervals are represented, and (3) the subjects had different histories of experience on intervening stimulus conditions (see Table 1). Similarities in variance across all three of these differences are shown in Table 2 for subjects at equivalent levels of practice. Only those subjects on the baseline condition during the sessions tabled are compared. It is clear from these results that, with similar amounts of practice, response latency variances exhibit minimal differences both between individuals and across a moderate range of $P_{1} P_{2}$ intervals. Thus, asymptotic performance is independent of mean synchronization latency for the intervals tested (cf. Kristofferson, 1976), and so is rate of acquisition.

\section{Shape of Response Latency Distributions}

Performance similarities at different $\mathbf{P}_{\mathbf{1}} \mathbf{P}_{\mathbf{2}}$ intervals are evident not only in terms of variances but also in terms of the shape of response latency distributions, as shown in Figure 3. This figure displays relative frequency distributions of response latencies, using $5 \mathrm{msec}$ bins, for subject G.H. at $460 \mathrm{msec}$ and for subject G.T. at $310 \mathrm{msec}$. Each distribution represents five sessions, or 1,500 responses, at equivalent levels of practice. However, antecedent history and sequence of synchronization training are different for the two subjects, as shown in Table 1. Both distributions are very symmetrical and sharppeaked with short tails. All responses fall within a 50 -msec time window centered about the time point corresponding to $P_{2}$ onset. Ninety percent of the response latencies fall within a $24 \mathrm{msec}$ time window.

Mean within-block variances for subjects G.T. and G.H. are $54.1 \mathrm{msec}^{2}$ and $51.2 \mathrm{msec}^{2}$, respectively, while corresponding total variances are 56.8 and $51.8 \mathrm{msec}^{2}$. The small discrepancy between mean within-block variance and total variance indicates the data are stable and that changes in responding between blocks and between sessions are negligible. The mean response latency for each distribution is

Table 2

Response Latency Variances at Several Levels of Practice

\begin{tabular}{|c|c|c|c|}
\hline Sessions & Subject & Variance & $P_{1} P_{2}$ Interval \\
\hline $101-105$ & $\begin{array}{l}\text { M.W. } \\
\text { J.B. }\end{array}$ & $\begin{array}{l}114.9 \\
119.8\end{array}$ & $\begin{array}{l}360 \\
460\end{array}$ \\
\hline $121-125$ & $\begin{array}{l}\text { G.T. } \\
\text { C.H. } \\
\text { J.B. }\end{array}$ & $\begin{array}{l}73.6 \\
72.3 \\
84.7\end{array}$ & $\begin{array}{l}310 \\
310 \\
460\end{array}$ \\
\hline $176-180$ & $\begin{array}{l}\text { G.T. } \\
\text { G.H. }\end{array}$ & $\begin{array}{l}54.1 \\
51.2\end{array}$ & $\begin{array}{l}310 \\
460\end{array}$ \\
\hline
\end{tabular}


$307.5 \mathrm{msec}$ for subject G.T. and $460.8 \mathrm{msec}$ for subject G.H. while the ratio of standard deviation to mean is .024 for subject G.T., at a $P_{1} P_{2}$ interval of $310 \mathrm{msec}$, and .016 for subject G.H. at $460 \mathrm{msec}$. It is clear that standard deviation is not a constant proportion of the mean in this task. Furthermore, these ratios are much lower than the estimates of .060 to .100 obtained by Getty (1975) for duration discrimination and by Woodrow $(1930,1933)$ for time estimation. The lowest previous estimate of the ratio between standard deviation of response latencies and mean latency is .023 , obtained by Wing and Kristofferson (1973) using interresponse times. The important point is that this ratio is not constant in synchronization performance, at least over the range of $P_{1} P_{2}$ intervals tested here. Absolute performance level is constant, such that the ratio of standard deviation to mean response latency becomes increasingly small the longer the synchronization interval.

\section{Low Variances}

Low variances, defined as the lowest mean withinblock variance obtained for a group of five sessions under the baseline condition $\left(\mathbf{P}_{1} \mathbf{P}_{2}\right)$, are shown in Table 3 for each subject. Effects of practice are evident across subjects and seemingly independent of the mean, as discussed earlier. All response latency variances tabled are well below earlier estimates of minimum S-R latency variance from RT studies. The lowest value of $46.2 \mathrm{msec}^{2}$ even reduces the minimum S-R latency variance obtained by Kristofferson (1976), using a synchronization task, by a factor of 2 . Average mean error of synchrony, neglecting sign, associated with these low variances is $1.18 \mathrm{msec}$ with the largest being $2.5 \mathrm{msec}$. The standard deviations of session means were generally small, with a mean of $1.2 \mathrm{msec}$, providing further evidence of the stability of performance in this task.

Figure 4 shows two low-variance response latency distributions obtained for subject G.H. One distribution is based on five sessions of synchronization performance under the baseline condition, in which the foreperiod is variable, and the other represents five sessions of responding with a constant foreperiod. It is obvious that any effect of foreperiod variability is negligible in terms of the mean, variance

Table 3

Low Variances Obtained During Subject-Paced Synchronization

\begin{tabular}{lcccr}
$\begin{array}{l}\text { Sub- } \\
\text { ject }\end{array}$ & Sessions & $\begin{array}{c}\text { Mean } \\
(\mathrm{msec})\end{array}$ & $\begin{array}{c}\text { Variance } \\
\left(\mathrm{msec}^{2}\right)\end{array}$ & SE \\
\hline M.W. & $101-105$ & 360.2 & 114.9 & 2.56 \\
J.B. & $121-125$ & 460.4 & 84.7 & 1.13 \\
C.H. & $121-125$ & 308.5 & 72.3 & .52 \\
G.T. & $176-180$ & 307.5 & 54.1 & .90 \\
G.H. & $206-210$ & 461.3 & 46.2 & .95 \\
\hline
\end{tabular}

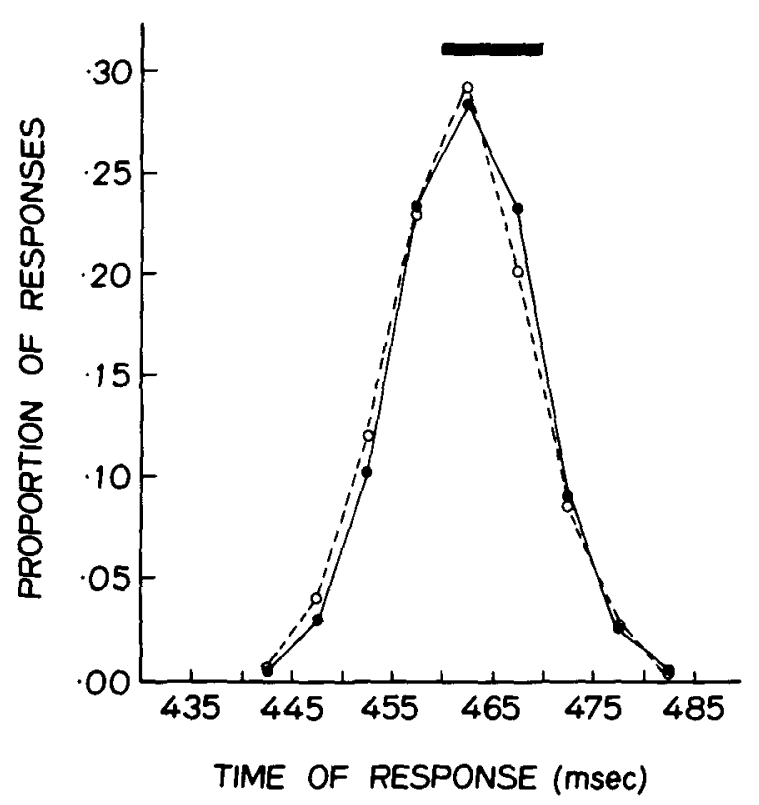

Figure 4. Low-variance relative frequency distributions, of 1,500 response latencies each, for subject G.H. for two foreperiod variability conditions with $a P_{1} P_{2}$ interval of $460 \mathrm{msec}$. Solid line: $M=462$, total variance $=45$, mean within-block variance $=44$, constant foreperiod. Dashed line: $M=461$, total variance $=48$, mean within-block variance $=46$, variable foreperiod. Rectangle marks time of occurrence of $P_{2}$.

and/or shape of the response latency distribution at these high levels of performance. The baseline distribution has a mean of $461.2 \mathrm{msec}$ and a mean withinblock variance of $46.2 \mathrm{msec}^{2}$ while the constant foreperiod distribution has a mean of 461.8 and a variance of $44.3 \mathrm{msec}^{2}$. Total variances are 47.6 and $45.1 \mathrm{msec}^{2}$ respectively, indicating again that changes between blocks and between sessions contribute little to the variance of the overall distribution. The error of synchrony is less than $2 \mathrm{msec}$ with the mode occurring just slightly after $P_{2}$ onset. All 1,500 responses fall within a $45 \mathrm{msec}$ time window, $90 \%$ within a 22 msec window, and $50 \%$ of all responses occur within $\pm 5 \mathrm{msec}$ of the median. The standard deviations of the distributions are both less than $1.5 \%$ of the mean.

These low-variance distributions are highly symmetrical with no indication that this shape is affected in any way by the placement of $\mathbf{P}_{2}$. The distributions are sharp-peaked, with straight sides and narrow skirts. They could be well described by the convolution of an isosceles triangle with a base of $32 \mathrm{msec}$ combined with a low-variance component which is normally distributed (cf. Kristofferson, 1976; Kristofferson \& Allan, 1973).

\section{$\mathbf{P}_{2}$ Only}

The initial auditory pulse, $P_{1}$, was removed from the stimulus sequence in order to ascertain the role of 
$P_{1}$ in controlling the timing of the synchronization responses. It should be noted that subjects were unaware of the existence of a foreperiod. Their subjective impression was that $\mathbf{P}_{\mathbf{1}}$ was generated instantaneously by their initiation response. This illusion was maintained even with the constant foreperiod, in which case the delay between $R_{i}$ and $P_{1}$ was 91 msec. Under the " $P_{2}$ only" condition, due to technical considerations, response latencies and synchronization intervals were still measured relative to the time-point $T_{0}$ (see Figure 1), corresponding to $P_{1}$ onset if it were present. This time-point is not defined for the subject under this condition. Thus, if timing control of $R_{S}$ were transferred from $P_{1}$ to some aspect of $R_{i}$, not only would the effective synchronization interval be increased, by an amount equal to the foreperiod duration, but latency variance would also include a component equal to the variability of the foreperiod.

The results of $P_{1}$ removal are shown in Table 4. Subjects experienced this manipulation at different points in practice, accounting for the range of values tabled under baseline variance. The interesting result is that differences in response latency variance between " $P_{2}$ only" and baseline $\left(P_{1} P_{2}\right)$ sessions are remarkably similar. These differences, with a variable foreperiod, range from 30.7 to $38.1 \mathrm{msec}^{2}$ the mean difference being only $1.1 \mathrm{msec}^{2}$ greater than that predicted by the simple addition of mean baseline variance with the experimentally induced component of variance $\left(33.3 \mathrm{msec}^{2}\right)$ associated with the variable foreperiod. With a constant foreperiod used in the " $P_{2}$ only" condition, baseline and " $P_{2}$ only" variances do not differ. These results indicate that when $P_{1}$ is present, it controls timing of the synchronization response; but when $P_{1}$ is absent, the control of timing can be transferred, all but perfectly, to some aspect of the initiation response. Any changes in performance observed following this transfer of control can be accounted for by the additional com-

Table 4

Comparison of Performance with the Baseline $\left(P_{1} P_{2}\right)$ Condition vs. Performance with the " $P_{2}$ Only" and the Constant Foreperiod " $P_{2}$ Only" Conditions

\begin{tabular}{lccc}
\hline \multicolumn{4}{c}{ Variance } \\
\cline { 3 - 4 } Subject & Condition & Baseline & Difference \\
\hline & \multicolumn{3}{c}{ "P $_{2}$ Only" Condition } \\
G.H. & 100.4 & 64.8 & 35.6 \\
G.T. & 84.8 & 54.1 & 30.7 \\
C.H. & 105.3 & 72.3 & 33.0 \\
M.W. & 153.1 & 115.0 & 38.1 \\
& "P & Only" Condition (Constant FP) \\
C.H. & 70.4 & 72.3 & -1.9 \\
M.W. & 117.2 & 115.0 & 2.2 \\
\hline
\end{tabular}

Note-Variances are expressed in milliseconds ${ }^{2}$. Average difference for the first condition was 34.4 and for the second was. 15 .

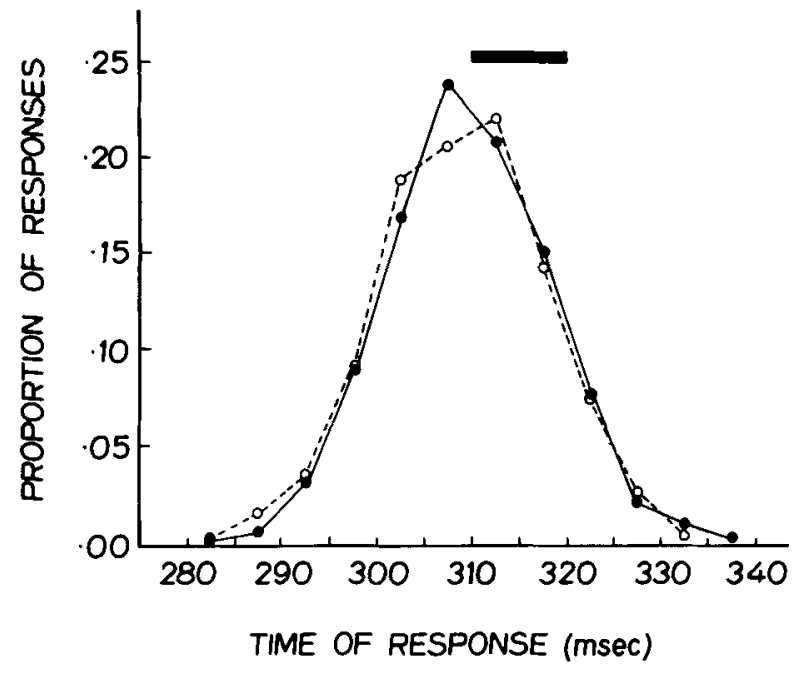

Figure 5. Frequency distributions, of 1,500 response latencies each, for subject $C . H$. under " $P_{2}$ only" with constant foreperiod (solid) and baseline (dashed) conditions with a $P_{1} P_{2}$ interval of 310 msec. Both distributions have a mean of 309 msec and mean within-block variance of 72 msec $^{2}$.

ponent of foreperiod variability which is added into the response latency measurements.

As mentioned earlier, the transfer of control to some aspect of $R_{i}$, under the " $P_{2}$ only" condition, entails increasing the effective synchronization interval by an amount equal to the foreperiod duration. The additional delay required for synchronization with a variable foreperiod averages $61 \mathrm{msec}$ while that required with a constant foreperiod is $91 \mathrm{msec}$. It is interesting to note that, in spite of these increases in the effective synchronization interval, mean response latency was unchanged even during the first block of trials following the transition. Thus, the internally timed delays, presumably underlying synchronization performance, appear to be quickly and accurately adjustable. Earlier work (Kristofferson, 1976) had shown that 10 -msec changes in the $P_{1} P_{2}$ interval could be accommodated with very little deterioration of performance. In the present instance, however, changes in the synchronization interval of almost $100 \mathrm{msec}$ are easily accommodated as shown in Figure 5 by the similarity in response latency distributions obtained for subject C.H. With the exception of the flattened peak on the baseline $\left(\mathrm{P}_{1} \mathrm{P}_{2}\right)$ distribution, the two plots are nearly superimposed. The baseline distribution has a mean of $308.5 \mathrm{msec}$ and a variance of $72.3 \mathrm{msec}^{2}$ relative to a mean of 309.1 msec and variance of $71.6 \mathrm{msec}^{2}$ for the other distribution.

\section{Sequential Dependencies}

Early in acquisition, subjects exhibited a strong positive dependency between successive response latencies such that perseveration of early or late 
responding over several trials was quite common. However, as practice continued, this positive dependency diminished to the point where no evidence of a temporal autocorrelation between successive response latencies remained. This independence of response latencies is shown in Figure 6. The upper two panels display response latency distributions and lag 1 autocorrelation analyses for subjects G.H. and G.T. obtained under the baseline condition, while the lower panels display similar data obtained under the " $P_{2}$ only" condition. In all cases, time of response on trial $(n+1)$ is independent of the response latency on trial $\mathrm{n}$, as indicated by the flat autocorrelation plots.

Under the " $\mathrm{P}_{2}$ only" condition with a variable foreperiod, the subject is in effect timing his synchronization response from a time-point that has some temporal variability associated with it relative to the time of $P_{2}$ onset. This means that feedback under these conditions is not always appropriate. That is, for a given accurately timed, internal delay, the feedback signal will vary as a function of the

SUBJECT GH
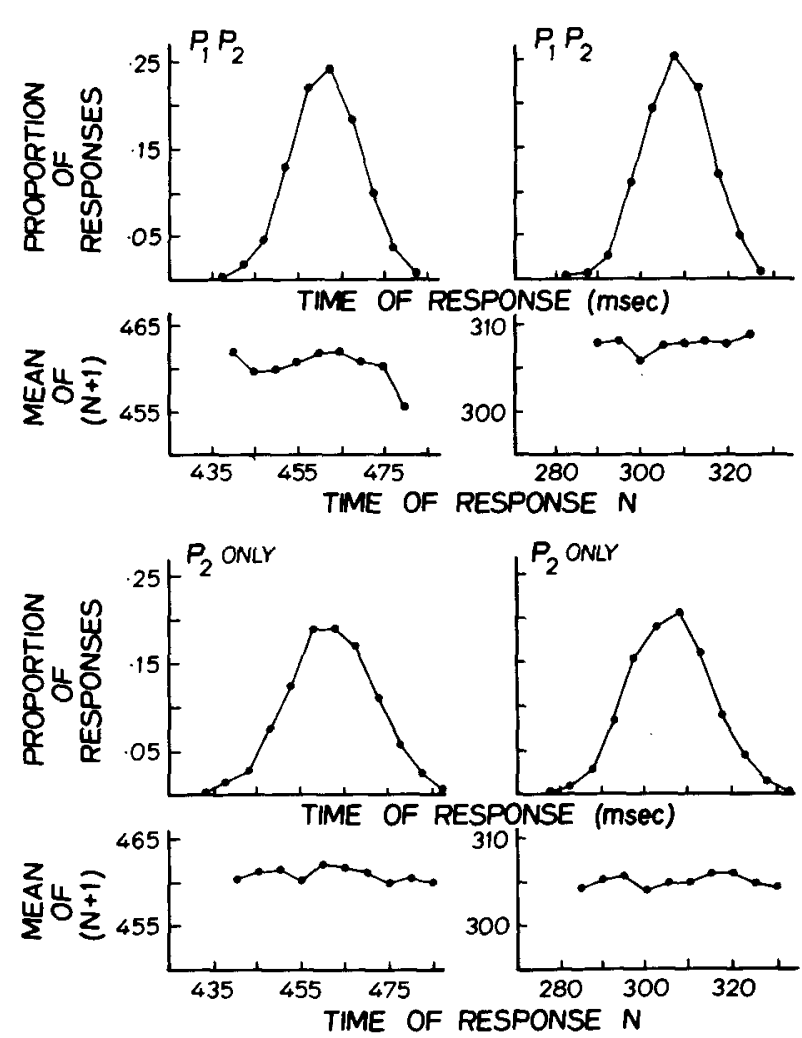

Figure 6. Lag 1 autocorrelation analyses, of 1,500 response latencies each, for subjects G.H. and G.T. under two different conditions. Top figures in each of the four panels show total distribution of response latencies. Lower figures show the mean on trial $(n+1)$, given that the response on trial $n$ was of the latency on the abscissa. foreperiod duration. If such erroneous information were taken into account by the subject on each trial, response latency variances associated with the " $P_{2}$ only" condition would be expected to be inflated beyond the levels observed, which are simply the addition of baseline and foreperiod variance components. The failure to find this additional increase in variance, in conjunction with the finding of no autocorrelation between response latencies at this level of practice, suggests that feedback is being ignored, at least on a trial-to-trial basis, although some averaging of feedback signals may be taking place over many trials. Such averaging could provide reliable information for maintenance of the internallytimed delay required for accurate synchronization.

\section{Additional Stimulus Manipulations.}

Removal of $P_{1}$ had little effect on synchronization performance. However, the same was not true when $P_{2}$ was removed. For subjects G.H., C.H., and J.B., performance was severely disrupted immediately upon transfer to the " $P_{1}$ only" condition. Response latency variances increased three to four times over baseline $\left(\mathbf{P}_{1} \mathbf{P}_{2}\right)$ levels. Continued practice under this condition was typically characterized by further degradation of performance followed by gradual improvement. Subject G.T. was the exception and exhibited little difficulty with this condition. After only 20 sessions, his performance was quite stable. Mean response latency variance for the last five sessions under the " $P_{1}$ only" condition was $95.5 \mathrm{msec}^{2}$ relative to a postcondition baseline variance of $63.0 \mathrm{msec}^{2}$.

Improvements in performance for the other subjects were extremely slow and frustrating. Consequently, only subject G.H. persevered until a stable level of performance was obtained. This required 55 sessions, at which point his mean latency variance was $92.5 \mathrm{msec}^{2}$ relative to a postcondition baseline variance of $58.5 \mathrm{msec}^{2}$. The difference in variance is $34 \mathrm{msec}^{2}$. A similar value of $32.5 \mathrm{msec}^{2}$ was obtained for subject G.T. Since these values were so close to the variance differences observed between baseline and " $P_{2}$ only" conditions, it was thought that subjects under the " $P_{1}$ only" condition might simply be ignoring $P_{1}$, in the absence of $P_{2}$, such that the additional variance observed could be accounted for by the subject timing from the initiation response with its associated $33.3 \mathrm{msec}^{2}$ foreperiod variability. To test this idea, the foreperiod was made constant for subject G.H. No change in performance occurred. Mean response latency variance for the last five sessions with $\mathrm{P}_{1}$ only and a constant foreperiod was $92.3 \mathrm{msec}^{2}$. Furthermore, when both $P_{1}$ and $P_{2}$ were removed from the stimulus sequence for G.T., the difference in observed variance, relative to baseline, increased to 60.3 from $32.5 \mathrm{msec}^{2}$ suggesting that the effect of $P_{1}$ removal is independent and additive to the effect of $P_{2}$ removal. However, the nature 
of the effect caused by deletion of $\mathrm{P}_{2}$ is unclear. It may simply serve some immediate, perceptual feedback role which cannot be totally compensated for by the delayed feedback.

\section{GENERAL DISCUSSION}

These results clearly demonstrate that, with special procedures, the upper bound on minimum S-R latency variance can be reduced substantially below levels provided by previous research. Response latency variance values of less than $50 \mathrm{msec}^{2}$ represent a reduction in variance by a factor of 2 over those values obtained by Kristofferson (1976), indicating that the changes in procedure incorporated into the present experiments were instrumental in reducing variances further. However, it is not clear which of the several changes were responsible for this improvement.

The finding of such low variances, in conjunction with the observation of continuing improvements in performance even after 80,000 trials (see Figure 2), raises an important question. Is there a lower limit on variance which is greater than zero? Based on the current data, no definitive answer can be made. The curves in Figure 2 may be slowly approaching zero variance and the limit may be completely deterministic timing.

Whatever the ultimate limit, the present results support Kristofferson's (1976) proposal for the existence of deterministic internal delays; that is, adjustable delays within the processing system which can be inserted into $S-R$ chains without any associated decrease in the temporal certainty of the response. However, not only is the variance of response latencies independent of the mean latency, but so is the rate of acquisition, which indicates that longer delays are no more difficult to establish than shorter ones. This is true over a range of synchronization intervals from 310 to $550 \mathrm{msec}$, if the constant foreperiod data are taken into account, which corresponds to Region II in Kristofferson's experiments.

Information regarding the locus of these internally timed delays is not directly forthcoming from this experiment, but some of the data suggest further speculation. Kristofferson (1976) has proposed that deterministic delays are contained in the afferent latency of the stimulus. The " $\mathrm{P}_{2}$ only" results can be interpreted as providing some additional support for this notion, or at least for the notion of zerovariance afferent latencies. Transfer of timing control for synchronization responding from auditory to tactile stimulation had no effect on variance measures of performance. In order to obtain such a result, if afferent latencies were variable, one would have to assume that the afferent latencies associated with auditory and tactile stimuli have exactly the same variability. Such an assumption does not seem to be as parsimonious as simply assuming zero variance.

Is it possible that the control of timing of the synchronization response could be accomplished by something other than the central sensory effects of the initiation response and, therefore, nullify such reasoning? It does not appear so. There are only two possible strategies for this timing control: use of the time-point associated with central registration of the tactile stimulation following the response or of that associated with the central response trigger preceding the response. With a constant foreperiod and use of the first strategy, the time-point associated with initiation of central timing is perfectly timelocked (assuming zero-variance afferent latencies) to the electronic switching involved with initiation of the $P_{1} P_{2}$ interval such that the delay between commencement of objective and subjective timing is constant. However, such is not the case for the second type of timing control. The time-point associated with the central response trigger has some temporal variability with respect to the time-point of the electronic switching produced by the initiation response due to variability of the efferent delay separating these two events. Thus, use of the response trigger for timing would entail the addition of another variance component into the observed response latency distribution. If one assumes this variance to be of the same order of magnitude as the estimate provided by Wing and Kristofferson (1973), then the second possibility for timing control must be rejected based on the finding of no increase in variance under the " $P_{2}$ only" condition.

Once established, synchronization performance is remarkably unaffected by major changes in procedure. Subjects can accommodate alterations in the synchronization interval of nearly $100 \mathrm{msec}$ and are able to transfer the control of synchronization timing from an auditory to a tactile stimulus. The fact that cross-modal transfers of this nature are possible in this task suggests that the internally timed delays underlying synchronization performance might serve a quite general and useful purpose in acts of information processing within the central nervous system.

A problem is: How are these accurate, internally timed delays established and maintained? It is clear that some form of feedback is being effectively used early in acquisition because mean response latencies center on the point of veridical synchrony almost right from the start. Yet is also evident that, later in practice, response latencies are independent of feedback, at least on a trial-to-trial basis. This suggests that the accuracy of the internally timed interval is maintained by an averaging process that integrates feedback information over a large number of trials. If it is further assumed that the sample size increases 
with practice, such a strategy could explain many of the sequential dependency results obtained. With a small sample size, the standard error of the mean is large. Therefore, early in practice, strong positive dependencies between response latencies would be expected due to a slow wandering of the mean during a session. However, with a large sample of feedback information, the standard error of the mean would be quite small, resulting in stable responding from block-to-block and session-to-session, as observed.

There are two types of feedback information available to the subject: the delayed feedback provided by the experimenter, and the immediate perceptual feedback, derived from the temporal relation between the central sensory effects caused by $P_{2}$ and those of the subject's synchronization response. The question concerning how these sources of information are used is not answered yet, but is currently under investigation. Preliminary results suggest that the delayed feedback is useful for helping the subject interpret immediate perceptual feedback information during early acquisition but becomes redundant later in practice. It can be removed from the stimulus sequence with no detrimental effects on performance. The delayed feedback signal can serve as a surrogate for $P_{2}$, as in the " $P_{1}$ only" condition, but not without some decrement in performance. Thus, $P_{2}$ seems to serve the crucial feedback role in maintaining the accuracy of synchronization responses.

Much of this discussion has been speculative. However, it appears that the use of exhaustive training in conjunction with an experimental procedure that allows manipulation of stimulus events within the response-stimulus synchronization task provides a useful tool for theorizing about elementary timing mechanisms.

\section{REFERENCES}

Allan, L. G. The relationship between judgments of successiveness and judgments of order. Perception \& Psychophysics, 1975, 18, 29-36.

Allan, L. G., \& Kristofferson, A. B. Successiveness discrimination: Two models. Perception \& Psychophysics, 1974, 15, $37-46$.

GetTy, D. J. Discrimination of short temporal intervals: A comparison of two models. Perception \& Psychophysics, 1975, 18, $1-8$.

Kornblum, S. Simple reaction time as a race between signal detection and time estimation: A paradigm and model. Perception \& Psychophysics, 1973, 13, 108-112.

Kristofferson, A. B. Attention and psychophysical time. Acta Psychologica, 1967, 27, 93-100.

Kristofferson, A. B. Low-variance stimulus-response latencies: Deterministic internal delays? Perception \& Psychophysics, 1976, 20, 89-100.

Kristofferson, A. B. A real-time criterion theory of duration discrimination. Perception \& Psychophysics, 1977, 21, 105-117.

Kristofferson, A. B., \& Allan, L. G. Successiveness and duration discrimination. In S. Kornblum (Ed.), Attention and performance IV. New York and London: Academic Press, 1973.

Naatanen, R., Muranen, V., \& Merisalo, A. Timing of the expectancy peak in simple reaction time situation. Acta Psychologica, 1974, 38, 461-470.

Ollman, R. T., \& Billington, M. J. The deadline model for simple reaction times. Cognitive Psychology, 1972, 3, 311-336.

Sasıow, C. A. Dual payoff band control of reaction time. Perception \& Psychophysics, 1974, 15, 108-114.

Snovgrass, J. G. Foreperiod effects in simple reaction time: Anticipation or expectancy? Journal of Experimental Psychology Monograph, 1969, 79(3, Part 2), 1-19.

Snodgrass, J. G., Luce, R. D., \& Galanter, E. Some experiments on simple and choice reaction time. Journal of Experimental Psychology, 1967, 75, 1-17.

Wing, A. M., \& Kristofferson, A. B. Response delays and the timing of discrete motor responses. Perception and Psychophysics, 1973, 14, 5-12.

Woovrow, H. The reproduction of temporal intervals. Journal of Experimental Psychology, 1930, 13, 473-499.

Woovrow, H. Individual differences in the reproduction of temporal intervals. American Journal of Psychology, 1933, 45, 271-281.

(Received for publication October 30, 1979; revision accepted December $18,1979$. 